A NNALES

UNIVERSITATIS MARIAE CURIE-SKŁODOWSKA

LUBLIN - POLONIA

VOL. LXVI, NO. 1, 2012

SECTIO A

$13-23$

B. A. FRASIN

\title{
On certain general integral operators of analytic functions
}

\begin{abstract}
In this paper, we obtain new sufficient conditions for the operators $F_{\alpha_{1}, \alpha_{2}, \ldots, \alpha_{n}, \beta}(z)$ and $G_{\alpha_{1}, \alpha_{2}, \ldots, \alpha_{n}, \beta}(z)$ to be univalent in the open unit disc $\mathcal{U}$, where the functions $f_{1}, f_{2}, \ldots, f_{n}$ belong to the classes $\mathcal{S}^{\star}(a, b)$ and $\mathcal{K}(a, b)$. The order of convexity for the operators $F_{\alpha_{1}, \alpha_{2}, \ldots, \alpha_{n}, \beta}(z)$ and $G_{\alpha_{1}, \alpha_{2}, \ldots, \alpha_{n}, \beta}(z)$ is also determined. Furthermore, and for $\beta=1$, we obtain sufficient conditions for the operators $F_{n}(z)$ and $G_{n}(z)$ to be in the class $\mathcal{K}(a, b)$. Several corollaries and consequences of the main results are also considered.
\end{abstract}

1. Introduction and definitions. Let $\mathcal{A}$ denote the class of functions of the form

$$
f(z)=z+\sum_{n=2}^{\infty} a_{n} z^{n}
$$

which are analytic in the open unit $\operatorname{disc} \mathcal{U}=\{z:|z|<1\}$. Further, by $\mathcal{S}$ we shall denote the class of all functions in $\mathcal{A}$ which are univalent in $\mathcal{U}$. A function $f(z) \in \mathcal{A}$ is said to be starlike of order $\gamma(0 \leq \gamma<1)$ if it satisfies

$$
\operatorname{Re}\left(\frac{z f^{\prime}(z)}{f(z)}\right)>\gamma \quad(z \in \mathcal{U})
$$

1991 Mathematics Subject Classification. 30C45.

Key words and phrases. Analytic functions, starlike and convex functions, integral operator. 
Also, we say that a function $f(z) \in \mathcal{A}$ is said to be convex of order $\gamma(0 \leq$ $\gamma<1)$ if it satisfies

$$
\operatorname{Re}\left(1+\frac{z f^{\prime \prime}(z)}{f^{\prime}(z)}\right)>\gamma \quad(z \in \mathcal{U}) .
$$

We denote by $\mathcal{S}^{\star}(\gamma)$ and $\mathcal{K}(\gamma)$, respectively, the usual classes of starlike and convex functions of order $\gamma(0 \leq \gamma<1)$ in $\mathcal{U}$.

A function $f \in \mathcal{A}$ is said to be in the class $\mathcal{S}^{\star}(a, b)$ if

$$
\left|\frac{z f^{\prime}(z)}{f(z)}-a\right|<b \quad(z \in \mathcal{U} ;|a-1|<b \leq a)
$$

and a function $f \in \mathcal{A}$ is said to be in the class $\mathcal{K}(a, b)$ if

$$
\left|1+\frac{z f^{\prime \prime}(z)}{f^{\prime}(z)}-a\right|<b \quad(z \in \mathcal{U} ;|a-1|<b \leq a) .
$$

From (1.3) and (1.4), we have

$$
\operatorname{Re}\left(\frac{z f^{\prime}(z)}{f(z)}\right)>a-b \quad(z \in \mathcal{U} ;|a-1|<b \leq a)
$$

and

$$
\operatorname{Re}\left(1+\frac{z f^{\prime \prime}(z)}{f^{\prime}(z)}\right)>a-b \quad(z \in \mathcal{U} ;|a-1|<b \leq a) .
$$

The class $\mathcal{S}^{\star}(a, b)$ was introduced by Jakubowski [12]. It is clear that $a>\frac{1}{2}$, $\mathcal{S}^{\star}(a, b) \subset \mathcal{S}^{\star}(a-b) \subset \mathcal{S}^{\star}(0) \equiv \mathcal{S}^{\star}$ and $\mathcal{K}(a, b) \subset \mathcal{K}(a-b) \subset \mathcal{K}(0) \equiv \mathcal{K}$. Further, applying the Briot-Bouquet differential subordination [9], we can easily see that $\mathcal{K}(a, b) \subset \mathcal{S}^{\star}(a, b)$.

Several authors (e.g., see $[4,5,6,8,10,11,15,16]$ ), obtained many sufficient conditions for the univalency of the integral operators

$$
F_{\alpha_{1}, \alpha_{2}, \ldots, \alpha_{n}, \beta}(z)=\left\{\beta \int_{0}^{z} t^{\beta-1} \prod_{i=1}^{n}\left(\frac{f_{i}(t)}{t}\right)^{\alpha_{i}} d t\right\}^{\frac{1}{\beta}}
$$

and

$$
G_{\alpha_{1}, \alpha_{2}, \ldots, \alpha_{n}, \beta}(z)=\left\{\beta \int_{0}^{z} t^{\beta-1} \prod_{i=1}^{n}\left(f_{i}^{\prime}(t)\right)^{\alpha_{i}} d t\right\}^{\frac{1}{\beta}},
$$

where the functions $f_{1}, f_{2}, \ldots, f_{n}$ belong to the class $\mathcal{A}$ and the parameters $\alpha_{1}, \alpha_{2}, \ldots, \alpha_{n}$, and $\beta$ are complex numbers such that the integrals in (1.5) and (1.6) exist. Here and throughout in the sequel every many-valued function is taken with the principal branch. 
For $\beta=1$, we obtain the integral operators

$$
F_{n}(z)=\int_{0}^{z}\left(\frac{f_{1}(t)}{t}\right)^{\alpha_{1}} \ldots\left(\frac{f_{n}(t)}{t}\right)^{\alpha_{n}} d t
$$

and

$$
G_{n}(z)=\int_{0}^{z}\left(f_{1}^{\prime}(t)\right)^{\alpha_{1}} \ldots\left(f_{n}^{\prime}(t)\right)^{\alpha_{n}} d t
$$

introduced and studied by Breaz and Breaz [5] and Breaz et al. [7], respectively.

In this paper, we obtain new sufficient conditions for the operators $F_{\alpha_{1}, \alpha_{2}, \ldots, \alpha_{n}, \beta}(z)$ and $G_{\alpha_{1}, \alpha_{2}, \ldots, \alpha_{n}, \beta}(z)$ defined by (1.5) and (1.6) to be univalent in the open unit $\operatorname{disc} \mathcal{U}$, where the functions $f_{1}, f_{2}, \ldots, f_{n}$ belong to the above classes $\mathcal{S}^{\star}(a, b)$ and $\mathcal{K}(a, b)$. The order of convexity for the operators $F_{\alpha_{1}, \alpha_{2}, \ldots, \alpha_{n}, \beta}(z)$ and $G_{\alpha_{1}, \alpha_{2}, \ldots, \alpha_{n}, \beta}(z)$ is also determined. Furthermore, we obtain sufficient conditions for the operators $F_{n}(z)$ and $G_{n}(z)$ defined by (1.5) and (1.6) to be in the class $\mathcal{K}(a, b)$.

In the proofs of our main results we need the following univalence criteria. The first result, i.e. Lemma 1.1 is a generalization of the wellknown univalence criterion of Becker [2] (which in fact corresponds to the case $\beta=\delta=1$ ), while the second, i.e. Lemma 1.2 is a generalization of Ahlfors' and Becker's univalence criterion $[1,3]$ (which corresponds to the case $\beta=1$ ).

Lemma 1.1 ([13]). Let $\delta$ be a complex number with $\operatorname{Re}(\delta)>0$. If $f \in \mathcal{A}$ satisfies

$$
\frac{1-|z|^{2 \operatorname{Re}(\delta)}}{\operatorname{Re}(\delta)}\left|\frac{z f^{\prime \prime}(z)}{f^{\prime}(z)}\right| \leq 1,
$$

for all $z \in \mathcal{U}$, then, for any complex number $\beta$ with $\operatorname{Re}(\beta) \geq \operatorname{Re}(\delta)$, the integral operator

$$
F_{\beta}(z)=\left\{\beta \int_{0}^{z} t^{\beta-1} f^{\prime}(t) d t\right\}^{\frac{1}{\beta}}
$$

is in the class $\mathcal{S}$.

Lemma 1.2 ([14]). Let $\beta$ be a complex number with $\operatorname{Re}(\beta)>0$ and $c$ be a complex number with $|c| \leq 1, c \neq-1$. If $f \in \mathcal{A}$ satisfies

$$
\left.|c| z\right|^{2 \beta}+\left(1-|z|^{2 \beta}\right) \frac{z f^{\prime \prime}(z)}{\beta f^{\prime}(z)} \mid \leq 1
$$


for all $z \in \mathcal{U}$, then the integral operator

$$
F_{\beta}(z)=\left\{\beta \int_{0}^{z} t^{\beta-1} f^{\prime}(t) d t\right\}^{\frac{1}{\beta}}
$$

is in the class $\mathcal{S}$.

2. Univalence conditions for $\boldsymbol{F}_{\alpha_{1}, \alpha_{2}, \ldots, \alpha_{n}, \boldsymbol{\beta}}(\boldsymbol{z})$. We first prove

Theorem 2.1. Let $f_{i}(z) \in \mathcal{S}^{\star}\left(a_{i}, b_{i}\right) ;\left|a_{i}-1\right|<b_{i} \leq a_{i}, \alpha_{i} \in \mathbb{C}$ for all $i=1, \ldots, n$, and $\delta \in \mathbb{C}$ with

$$
\operatorname{Re}(\delta) \geq 2 \sum_{i=1}^{n}\left|\alpha_{i}\right| b_{i} .
$$

Then for any $\beta \in \mathbb{C}$ with $\operatorname{Re}(\beta) \geq \operatorname{Re}(\delta)$, the integral operator $F_{\alpha_{1}, \alpha_{2}, \ldots, \alpha_{n}, \beta}(z)$ defined by (1.5) is analytic and univalent in $\mathcal{U}$.

Proof. Defining

$$
h(z)=\int_{0}^{z} \prod_{i=1}^{n}\left(\frac{f_{i}(t)}{t}\right)^{\alpha_{i}} d t,
$$

we observe that $h(0)=h^{\prime}(0)-1=0$, where

$$
h^{\prime}(z)=\prod_{i=1}^{n}\left(\frac{f_{i}(z)}{z}\right)^{\alpha_{i}} .
$$

Differentiating both sides of (2.2) logarithmically, we obtain

$$
\frac{z h^{\prime \prime}(z)}{h^{\prime}(z)}=\sum_{i=1}^{n} \alpha_{i}\left(\frac{z f_{i}^{\prime}(z)}{f_{i}(z)}-1\right)
$$

which is equivalent to

$$
\frac{z h^{\prime \prime}(z)}{h^{\prime}(z)}=\sum_{i=1}^{n} \alpha_{i}\left(\frac{z f_{i}^{\prime}(z)}{f_{i}(z)}-a_{i}\right)+\sum_{i=1}^{n} \alpha_{i} a_{i}-\sum_{i=1}^{n} \alpha_{i} .
$$

Since $f_{i}(z) \in \mathcal{S}^{\star}\left(a_{i}, b_{i}\right) ;\left|a_{i}-1\right|<b_{i} \leq a_{i}$ for all $i=1,2, \ldots, n$, it follows from (2.3) that

$$
\begin{aligned}
\left|\frac{z h^{\prime \prime}(z)}{h^{\prime}(z)}\right| & \leq \sum_{i=1}^{n}\left|\alpha_{i}\right|\left|\frac{z f_{i}^{\prime}(z)}{f_{i}(z)}-a_{i}\right|+\sum_{i=1}^{n}\left|\alpha_{i}\right|\left|a_{i}-1\right| \\
& <2 \sum_{i=1}^{n}\left|\alpha_{i}\right| b_{i} .
\end{aligned}
$$


Multiplying both sides of (2.4) by $\frac{1-|z|^{2} \operatorname{Re}(\delta)}{\operatorname{Re}(\delta)}$ and making use of (2.1), we obtain

$$
\begin{aligned}
\frac{1-|z|^{2 \operatorname{Re}(\delta)}}{\operatorname{Re}(\delta)}\left|\frac{z h^{\prime \prime}(z)}{h^{\prime}(z)}\right| & \leq 2\left(\frac{1-|z|^{2 \operatorname{Re}(\delta)}}{\operatorname{Re}(\delta)}\right) \sum_{i=1}^{n}\left|\alpha_{i}\right| b_{i} \\
& <\frac{2}{\operatorname{Re}(\delta)} \sum_{i=1}^{n}\left|\alpha_{i}\right| b_{i} \leq 1 .
\end{aligned}
$$

Applying Lemma 1.1 for the function $h(z)$, we prove that $F_{\alpha_{1}, \alpha_{2}, \ldots, \alpha_{n}, \beta}(z) \in$ $\mathcal{S}$.

Letting $n=1, \alpha_{1}=\alpha, a_{1}=a, b_{1}=b$ and $f_{1}=f$ in Theorem 2.1, we have

Corollary 2.2. Let $f(z) \in \mathcal{S}^{\star}(a, b) ;|a-1|<b \leq a, \alpha \in \mathbb{C}$ and $\delta \in \mathbb{C}$ with $\operatorname{Re}(\delta)>2|\alpha| b$. Then for any $\beta \in \mathbb{C}$ with $\operatorname{Re}(\beta) \geq \operatorname{Re}(\delta)$, the integral operator

$$
F_{\alpha, \beta}(z)=\left\{\beta \int_{0}^{z} t^{\beta-1}\left(\frac{f(t)}{t}\right)^{\alpha} d t\right\}^{\frac{1}{\beta}}
$$

is analytic and univalent in $\mathcal{U}$.

Making use of Lemma 1.2, we prove the following theorem:

Theorem 2.3. Let $f_{i}(z) \in \mathcal{S}^{\star}\left(a_{i}, b_{i}\right) ;\left|a_{i}-1\right|<b_{i} \leq a_{i}, \alpha_{i} \in \mathbb{C}$ for all $i=1,2, \ldots, n$, and $\beta \in \mathbb{C}$ with

$$
\operatorname{Re}(\beta) \geq 2 \sum_{i=1}^{n}\left|\alpha_{i}\right| b_{i}
$$

and

$$
|c| \leq 1-\frac{2}{\operatorname{Re}(\beta)} \sum_{i=1}^{n}\left|\alpha_{i}\right| b_{i} \quad(c \in \mathbb{C}) .
$$

Then the integral operator $F_{\alpha_{1}, \alpha_{2}, \ldots, \alpha_{n}, \beta}(z)$ defined by (1.5) is analytic and univalent in $\mathcal{U}$. 
Proof. Let $f_{i}(z) \in \mathcal{S}^{\star}\left(a_{i}, b_{i}\right) ;\left|a_{i}-1\right|<b_{i} \leq a_{i}$ for all $i=1,2, \ldots, n$, it follows from (2.4) that

$$
\begin{aligned}
\left.|c| z\right|^{2 \beta}+\left(1-|z|^{2 \beta}\right) \frac{z h^{\prime \prime}(z)}{\beta h^{\prime}(z)} \mid & \leq|c|+\left|\frac{1-|z|^{2 \beta}}{\beta}\right|\left|\frac{z h^{\prime \prime}(z)}{h^{\prime}(z)}\right| \\
& \leq|c|+2\left|\frac{1-|z|^{2 \beta}}{\beta}\right| \sum_{i=1}^{n}\left|\alpha_{i}\right| b_{i} \\
& <|c|+\frac{2}{|\beta|} \sum_{i=1}^{n}\left|\alpha_{i}\right| b_{i} \\
& <|c|+\frac{2}{\operatorname{Re}(\beta)} \sum_{i=1}^{n}\left|\alpha_{i}\right| b_{i}
\end{aligned}
$$

which, in the light of the hypothesis (2.6), yields

$$
\left.|c| z\right|^{2 \beta}+\left(1-|z|^{2 \beta}\right) \frac{z h^{\prime \prime}(z)}{\beta h^{\prime}(z)} \mid \leq 1 .
$$

Finally, by applying Lemma 1.2 , we conclude that $F_{\alpha_{1}, \alpha_{2}, \ldots, \alpha_{n}, \beta}(z) \in \mathcal{S}$. have

Letting $n=1, \alpha_{1}=\alpha, a_{1}=a, b_{1}=b$ and $f_{1}=f$ in Theorem 2.3, we

Corollary 2.4. Let $f(z) \in \mathcal{S}^{\star}(a, b) ;|a-1|<b \leq a, \alpha \in \mathbb{C}$, and $\beta \in \mathbb{C}$ with

$$
\operatorname{Re}(\beta) \geq 2|\alpha| b
$$

and

$$
|c| \leq 1-\frac{2}{\operatorname{Re}(\beta)}|\alpha| b \quad(c \in \mathbb{C}) .
$$

Then the integral operator $F_{\alpha, \beta}(z)$ defined by (2.5) is analytic and univalent in $\mathcal{U}$.

3. Univalence conditions for $G_{\alpha_{1}, \alpha_{2}, \ldots, \alpha_{n}, \beta}(z)$. Now, we prove

Theorem 3.1. Let $f_{i}(z) \in \mathcal{K}\left(a_{i}, b_{i}\right) ;\left|a_{i}-1\right|<b_{i} \leq a_{i}, \alpha_{i} \in \mathbb{C}$ for all $i=1, \ldots, n$, and $\delta \in \mathbb{C}$ with

$$
\operatorname{Re}(\delta) \geq 2 \sum_{i=1}^{n}\left|\alpha_{i}\right| b_{i} .
$$

Then for any $\beta \in \mathbb{C}$ with $\operatorname{Re}(\beta) \geq \operatorname{Re}(\delta)$, the integral operator $G_{\alpha_{1}, \alpha_{2}, \ldots, \alpha_{n}, \beta}(z)$ defined by (1.6) is analytic and univalent in $\mathcal{U}$. 
Proof. Defining

$$
h(z)=\int_{0}^{z} \prod_{i=1}^{n}\left(f_{i}^{\prime}(t)\right)^{\alpha_{i}} d t
$$

we observe that $h(0)=h^{\prime}(0)-1=0$. On the other hand, it is easy to see that

$$
h^{\prime}(z)=\prod_{i=1}^{n}\left(f_{i}^{\prime}(z)\right)^{\alpha_{i}}
$$

Differentiating both sides of (3.1) logarithmically, we obtain

$$
\frac{z h^{\prime \prime}(z)}{h^{\prime}(z)}=\sum_{i=1}^{n} \alpha_{i}\left(\frac{z f_{i}^{\prime \prime}(z)}{f_{i}^{\prime}(z)}\right) \text {. }
$$

Thus, we have

$$
\frac{z h^{\prime \prime}(z)}{h^{\prime}(z)}=\sum_{i=1}^{n} \alpha_{i}\left(1+\frac{z f_{i}^{\prime \prime}(z)}{f_{i}^{\prime}(z)}-a_{i}\right)+\sum_{i=1}^{n} \alpha_{i}\left(a_{i}-1\right) .
$$

Let $f_{i}(z) \in \mathcal{K}\left(a_{i}, b_{i}\right) ;\left|a_{i}-1\right|<b_{i} \leq a_{i}$, for all $i=1,2, \ldots, n$, and following the same steps in the proof of Theorem 2.1, we get the required result.

Letting $n=1, \alpha_{1}=\alpha, a_{1}=a, b_{1}=b$ and $f_{1}=f$ in Theorem 3.1, we have

Corollary 3.2. Let $f(z) \in \mathcal{K}(a, b) ;|a-1|<b \leq a, \alpha$ and $\delta \in \mathbb{C}$ with $\operatorname{Re}(\delta) \geq 2|\alpha| b$. Then for any $\beta \in \mathbb{C}$ with $\operatorname{Re}(\beta) \geq \operatorname{Re}(\delta)$, the integral operator

$$
G_{\alpha, \beta}(z)=\left\{\beta \int_{0}^{z} t^{\beta-1}\left(f^{\prime}(t)\right)^{\alpha} d t\right\}^{\frac{1}{\beta}}
$$

is analytic and univalent in $\mathcal{U}$.

Using (3.1), (1.4) and applying Lemma 1.2, we prove the following theorem:

Theorem 3.3. Let $f_{i}(z) \in \mathcal{K}\left(a_{i}, b_{i}\right) ;\left|a_{i}-1\right|<b_{i} \leq a_{i}, \alpha_{i} \in \mathbb{C}$ for all $i=1, \ldots, n$ and $\beta \in \mathbb{C}$ with

$$
\operatorname{Re}(\beta) \geq 2 \sum_{i=1}^{n}\left|\alpha_{i}\right| b_{i}
$$

and

$$
|c| \leq 1-\frac{2}{\operatorname{Re}(\beta)} \sum_{i=1}^{n}\left|\alpha_{i}\right| b_{i} \quad(c \in \mathbb{C}) .
$$

Then the integral operator $G_{\alpha_{1}, \alpha_{2}, \ldots, \alpha_{n}, \beta}(z)$ defined by (1.6) is analytic and univalent in $\mathcal{U}$. 
Letting $n=1, \alpha_{1}=\alpha, a_{1}=a, b_{1}=b$ and $f_{1}=f$ in Theorem 3.3, we have

Corollary 3.4. Let $f(z) \in \mathcal{K}(a, b) ;|a-1|<b \leq a, \alpha$ and $\beta \in \mathbb{C}$ with

$$
\operatorname{Re}(\beta) \geq 2|\alpha| b
$$

and

$$
|c| \leq 1-\frac{2}{\operatorname{Re}(\beta)}|\alpha| b .
$$

Then the integral operator $G_{\alpha, \beta}(z)$ defined by (3.3) is analytic and univalent in $\mathcal{U}$.

4. Order of convexity. Now, we prove

Theorem 4.1. Let $f_{i}(z) \in \mathcal{S}^{\star}\left(a_{i}, b_{i}\right) ;\left|a_{i}-1\right|<b_{i} \leq a_{i}$, and $\alpha_{i}>0$ for all $i=1, \ldots, n$, with

$$
0 \leq 1-\sum_{i=1}^{n} \alpha_{i}\left(b_{i}+\frac{1}{2}\right)<1 \quad \text { and } \quad \sum_{i=1}^{n} \alpha_{i}\left(b_{i}+\frac{1}{2}\right) \leq 1 .
$$

Then the integral operator $F_{n}(z)$ defined by (1.7) is in the class

$$
\mathcal{K}\left(1-\sum_{i=1}^{n} \alpha_{i}\left(b_{i}+\frac{1}{2}\right)\right) \text {. }
$$

Proof. From (1.7), it follows that

$$
F_{n}^{\prime}(z)=\prod_{i=1}^{n}\left(\frac{f_{i}(z)}{z}\right)^{\alpha_{i}}
$$

Differentiating both sides of (4.1) logarithmically, we obtain

$$
1+\frac{z F_{n}^{\prime \prime}(z)}{F_{n}^{\prime}(z)}=\sum_{i=1}^{n} \alpha_{i}\left(\frac{z f_{i}^{\prime}(z)}{f_{i}(z)}\right)-\sum_{i=1}^{n} \alpha_{i}+1 .
$$

Since $f_{i}(z) \in \mathcal{S}^{\star}\left(a_{i}, b_{i}\right) ;\left|a_{i}-1\right|<b_{i} \leq a_{i}$ and $a_{i}>\frac{1}{2}$ for all $i=1,2, \ldots, n$, we have

$$
\begin{aligned}
\operatorname{Re}\left(1+\frac{z F_{n}^{\prime \prime}(z)}{F_{n}^{\prime}(z)}\right) & =\sum_{i=1}^{n} \alpha_{i} \operatorname{Re}\left(\frac{z f_{i}^{\prime}(z)}{f_{i}(z)}\right)-\sum_{i=1}^{n} \alpha_{i}+1 \\
& \geq \sum_{i=1}^{n} \alpha_{i}\left(a_{i}-b_{i}-1\right)+1 \\
& >1-\sum_{i=1}^{n} \alpha_{i}\left(b_{i}+\frac{1}{2}\right)
\end{aligned}
$$

Therefore, $F_{n}(z)$ is convex of order $1-\sum_{i=1}^{n} \alpha_{i}\left(b_{i}+\frac{1}{2}\right)$ in $\mathcal{U}$. 
Letting $n=1, \alpha_{1}=\alpha, a_{1}=a, b_{1}=b$ and $f_{1}=f$ in Theorem 4.1, we have

Corollary 4.2. Let $f(z) \in \mathcal{S}^{\star}(a, b) ;|a-1|<b \leq a$, and $\alpha>0$ with $0 \leq$ $1-\alpha\left(b+\frac{1}{2}\right)<1$ and $\alpha\left(b+\frac{1}{2}\right) \leq 1$. Then $\int_{0}^{z}\left(\frac{f(t)}{t}\right)^{\alpha} d t \in \mathcal{K}\left(1-\alpha\left(b+\frac{1}{2}\right)\right)$.

Next, we prove

Theorem 4.3. Let $f_{i}(z) \in \mathcal{K}\left(a_{i}, b_{i}\right) ;\left|a_{i}-1\right|<b_{i} \leq a_{i}$, and $\alpha_{i}>0$ for all $i=1, \ldots, n$, with

$$
0 \leq 1-\sum_{i=1}^{n} \alpha_{i}\left(b_{i}+\frac{1}{2}\right)<1 \quad \text { and } \quad \sum_{i=1}^{n} \alpha_{i}\left(b_{i}+\frac{1}{2}\right) \leq 1 .
$$

Then the integral operator $G_{n}(z)$ defined by $(1.8)$ is in the class

$$
\mathcal{K}\left(1-\sum_{i=1}^{n} \alpha_{i}\left(b_{i}+\frac{1}{2}\right)\right) .
$$

Proof. From (1.8), we have

$$
1+\frac{z G_{n}^{\prime \prime}(z)}{G_{n}^{\prime}(z)}=\sum_{i=1}^{n} \alpha_{i}\left(1+\frac{z f_{i}^{\prime \prime}(z)}{f_{i}^{\prime}(z)}\right)-\sum_{i=1}^{n} \alpha_{i}+1 .
$$

Let $f_{i}(z) \in \mathcal{K}\left(a_{i}, b_{i}\right) ;\left|a_{i}-1\right|<b_{i} \leq a_{i} ; a_{i}>\frac{1}{2}$ for all $i=1,2, \ldots, n$, and following the same steps in the proof of Theorem 4.1, we get the required result.

Letting $n=1, \alpha_{1}=\alpha, a_{1}=a, b_{1}=b$ and $f_{1}=f$ in Theorem 4.3, we have

Corollary 4.4. Let $f(z) \in \mathcal{K}(a, b) ;|a-1|<b \leq a$, and $\alpha>0$ with $0 \leq$ $1-\alpha\left(b+\frac{1}{2}\right)<1$ and $\alpha\left(b+\frac{1}{2}\right) \leq 1$. Then $\int_{0}^{z}\left(f^{\prime}(t)\right)^{\alpha} d t \in \mathcal{K}\left(1-\alpha\left(b+\frac{1}{2}\right)\right)$.

\section{Sufficient conditions for the operators $F_{n}(z)$ and $G_{n}(z)$.}

Theorem 5.1. Let $f_{i}(z) \in \mathcal{S}^{\star}\left(\gamma_{i}\right) ; 0 \leq \gamma_{i}<1$, for all $i=1,2, \ldots, n$. Then the integral operator $F_{n}(z)$ defined by $(1.7)$ is in the class $\mathcal{K}\left(a_{i}, b_{i}\right)$, where $a_{i}=\sum_{i=1}^{n} \alpha_{i} \gamma_{i}+1, b_{i}=\sum_{i=1}^{n} \alpha_{i}$ and $\sum_{i=1}^{n} \alpha_{i}\left(1-\gamma_{i}\right) \leq 1$ for all $i=1,2, \ldots, n$.

Proof. Let $f_{i}(z) \in \mathcal{S}^{\star}\left(\gamma_{i}\right) ; 0 \leq \gamma_{i}<1$, for all $i=1,2, \ldots, n$. Then it follows from (4.2) that

$$
\begin{aligned}
\operatorname{Re}\left(1+\frac{z F_{n}^{\prime \prime}(z)}{F_{n}^{\prime}(z)}\right) & =\sum_{i=1}^{n} \alpha_{i} \operatorname{Re}\left(\frac{z f_{i}^{\prime}(z)}{f_{i}(z)}\right)+1-\sum_{i=1}^{n} \alpha_{i} \\
& >\sum_{i=1}^{n} \alpha_{i} \gamma_{i}+1-\sum_{i=1}^{n} \alpha_{i}
\end{aligned}
$$


which proves that $F_{n}(z) \in \mathcal{K}\left(a_{i}, b_{i}\right)$, where $a_{i}=\sum_{i=1}^{n} \alpha_{i} \gamma_{i}+1$ and $b_{i}=$ $\sum_{i=1}^{n} \alpha_{i}$ for all $i=1,2, \ldots, n$.

Letting $n=1, \alpha_{1}=\alpha, \gamma_{1}=\gamma, a_{1}=a, b_{1}=b$ and $f_{1}=f$ in Theorem 5.1 , we have

Corollary 5.2. Let $f(z) \in \mathcal{S}^{\star}(\gamma) ; 0 \leq \gamma<1$. Then $\int_{0}^{z}\left(\frac{f(t)}{t}\right)^{\alpha} d t \in$ $\mathcal{K}(\alpha \gamma+1, \alpha)$, where $0<\alpha(1-\gamma) \leq 1$.

Using (4.3), we can prove the following theorem:

Theorem 5.3. Let $f_{i}(z) \in \mathcal{K}\left(\gamma_{i}\right) ; 0 \leq \gamma_{i}<1$, for all $i=1,2, \ldots, n$. Then the integral operator $G_{n}(z)$ defined by $(1.8)$ is in the class $\mathcal{K}\left(a_{i}, b_{i}\right)$, where $a_{i}=\sum_{i=1}^{n} \alpha_{i} \gamma_{i}+1, b_{i}=\sum_{i=1}^{n} \alpha_{i}$ and $\sum_{i=1}^{n} \alpha_{i}\left(1-\gamma_{i}\right) \leq 1$ for all $i=1,2, \ldots, n$.

Letting $n=1, \alpha_{1}=\alpha, \gamma_{1}=\gamma, a_{1}=a, b_{1}=b$ and $f_{1}=f$ in Theorem 5.3 , we have

Corollary 5.4. Let $f(z) \in \mathcal{K}(\gamma) ; 0 \leq \gamma<1$. Then $\int_{0}^{z}\left(f^{\prime}(t)\right)^{\alpha} d t \in \mathcal{K}(\alpha \gamma+$ $1, \alpha)$, where $0<\alpha(1-\gamma) \leq 1$.

\section{REFERENCES}

[1] Ahlfors, L. V., Sufficient conditions for quasiconformal extension, Discontinuous groups and Riemann surfaces (Proc. Conf., Univ. Maryland, College Park, Md., 1973), pp. 23-29. Ann. of Math. Studies, No. 79, Princeton Univ. Press, Princeton, N.J., 1974.

[2] Becker, J., Löwnersche Differentialgleichung und quasikonform fortsetzbare schlichte Funktionen, J. Reine Angew. Math. 255 (1972), 23-43.

[3] Becker, J., Löwnersche Differentialgleichung und Schlichtheitskriterien, Math. Ann. 202 (1973), 321-335.

[4] Breaz, D., Univalence properties for a general integral operator, Bull. Korean Math. Soc. 46 (2009), no. 3, 439-446.

[5] Breaz, D., Breaz, N., Two integral operators, Studia Universitatis Babeş-Bolyai Math., 47 (2002), no. 3, 13-19.

[6] Breaz, D., Breaz, N., Univalence conditions for certain integral operators, Studia Universitatis Babeş-Bolyai, Mathematica, 47 (2002), no. 2, 9-15.

[7] Breaz, D., Owa, S., Some extensions of univalent conditions for certain integral operators, Math. Inequal. Appl., 10 (2007), no. 2, 321-325.

[8] Bulut, S., Univalence preserving integral operators defined by generalized Al- Oboudi differential operators, An. Ştiinţ. Univ. "Ovidius" Constanţa Ser. Mat. 17 (2009), no. 1, 37-50.

[9] Eenigenburg, P., Miller, S. S., Mocanu, P. T. and Reade, M. O., On a Briot-Bouquet differential subordination, General inequalities, 3 (Oberwolfach, 1981), 339-348, Internat. Schriftenreihe Numer. Math., 64, Birkhäuser, Basel, 1983.

[10] Frasin, B. A., General integral operator defined by Hadamard product, Mat. Vesnik, 62 (2010), no. 2, 127-136.

[11] Frasin. B. A., Aouf, M. K., Univalence conditions for a new general integral operator, Hacet. J. Math. Stat., 39 (2010), no. 4, 567-575. 
[12] Jabkubowski, Z. J., On the coefficients of starlike functions of some classes, Ann. Polon. Math. 26 (1972), 305-313.

[13] Pascu, N., An improvement of Becker's univalence criterion, Proceedings of the Commemorative Session: Simion Stoïlow (Braşov, 1987), 43-48, Univ. Braşov, Braşov, 1987.

[14] Pescar, V., A new generalization of Ahlfor's and Becker's criterion of univalence, Bull. Malaysian Math. Soc. (2) 19 (1996), no. 2, 53-54.

[15] Seenivasagan, N., Sufficient conditions for univalence, Applied Math. E-Notes, 8 (2008), 30-35.

[16] Seenivasagan, N., Breaz, D., Certain sufficient conditions for univalence, Gen. Math. 15 (2007), no. 4, 7-15.

B. A. Frasin

Department of Mathematics

Al al-Bayt University

P.O. Box: 130095 Mafraq

Jordan

e-mail: bafrasin@yahoo.com

Received April 20, 2011 